OPEN ACCESS

Edited by:

Hyewon Phee,

Amgen, United States

Reviewed by:

Kristin Tarbell,

Amgen, United States

Herman Waldmann,

University of Oxford, United Kingdom

*Correspondence:

Parul Mehra

parul.mehra@bms.com

Andrew D. Wells

wellsa@chop.edu

Specialty section:

This article was submitted to Immunological Tolerance and Regulation,

a section of the journal

Frontiers in Immunology

Received: 24 November 2020

Accepted: 16 March 2021

Published: 15 April 2021

Citation:

Mehra P and Wells AD (2021) Variant to Gene Mapping to Discover New

Targets for Immune Tolerance.

Front. Immunol. 12:633219.

doi: 10.3389/fimmu.2021.633219

\section{Variant to Gene Mapping to Discover New Targets for Immune Tolerance}

\author{
Parul Mehra ${ }^{1 *}$ and Andrew D. Wells ${ }^{1,2 *}$ \\ ${ }^{1}$ Department of Pathology, The Children's Hospital of Philadelphia, Philadelphia, PA, United States, ${ }^{2}$ Department of Pathology and \\ Laboratory Medicine, Perelman School of Medicine, University of Pennsylvania, Philadelphia, PA, United States
}

The breakdown of immunological tolerance leads to autoimmune disease, and the mechanisms that maintain self-tolerance, especially in humans, are not fully understood. Genome-wide association studies (GWAS) have identified hundreds of human genetic loci statistically linked to autoimmune disease risk, and epigenetic modifications of DNA and chromatin at these loci have been associated with autoimmune disease risk. Because the vast majority of these signals are located far from genes, identifying causal variants, and their functional consequences on the correct effector genes, has been challenging. These limitations have hampered the translation of GWAS findings into novel drug targets and clinical interventions, but recent advances in understanding the spatial organization of the genome in the nucleus have offered mechanistic insights into gene regulation and answers to questions left open by GWAS. Here we discuss the potential for 'variant-to-gene mapping' approaches that integrate GWAS with 3D functional genomic data to identify human genes involved in the maintenance of tolerance.

Keywords: genome-wide association studies, single nucleotide polymorphism, autoimmunity, multi-omics, immune tolerance, variant-to-gene mapping

\section{INTRODUCTION}

Immune tolerance is established through clonal deletion during development of the immune repertoire, and is reinforced in the periphery through cell-intrinsic and -extrinsic mechanisms that limit activation and differentiation. Breakdown of central or peripheral tolerance can lead to autoimmunity (1), thus understanding the molecular and cellular mechanisms that control tolerance promises opportunities to therapeutically reprogram the immune system to treat inflammatory disease. The study of rare spontaneous or engineered monogenic mutations in the mouse have contributed significantly to our understanding of tolerance and autoimmunity. However, autoimmune disease in humans is relatively common, and the genetics of predisposition is complex, polygenic, and heavily influenced by environmental factors. Hundreds of genetic loci influencing susceptibility to various autoimmune and inflammatory disorders have been discovered by genome-wide association studies (GWAS) in humans, and in many cases have confirmed mouse models and yielded novel insights into mechanisms underpinning disease (2). Despite this, the identity of causal variants and their target genes remain largely unknown because variants rarely alter protein coding sequences. Instead, approximately $90 \%$ of immune diseaseassociated variants are located in non-coding regions, and integration of GWAS with cis-regulatory 
element annotations in immune cell types has shown that $\sim 60 \%$ map to immune cell enhancers (3-8). Identification of causal variants and their target genes are the next, necessary steps for understanding the molecular mechanisms by which genetic variation regulates immune tolerance, and for identifying new drug targets for treating autoimmune disease. This perspective discusses cellular and molecular mechanisms involved in the breakdown of immunological tolerance, as well as the potential of functional genomic approaches to define target genes in specific immune cell types to better understand the mechanism of autoimmunity.

\section{MAINTENANCE OF IMMUNOLOGIC TOLERANCE IN HUMANS AND ITS ROLE IN AUTOIMMUNITY}

Central tolerance takes place in the thymus and bone marrow through apoptotic deletion of autoreactive lymphocytes. Without negative selection, $\mathrm{T}$ and $\mathrm{B}$ cells respond to self-antigens and attack self-tissues, resulting in autoimmune pathologies (9-12). Autoreactive lymphocytes that escape negative selection in the primary lymphoid organs are normally held in check by additional, peripheral tolerance mechanisms that operate to dampen activation in secondary lymphoid tissues. The discovery of regulatory $\mathrm{T}$ cells (Tregs) represents a fundamental advance in our understanding the cellular basis of immune tolerance in the context of autoimmunity, transplantation, and cancer (13). The monogenic immune disorder IPEX (Immunodysregulation, Polyendocrinopathy, Entereopathy, X-linked syndrome) provides an example of a breach in tolerance in which mutations in the forkhead box P3 (FOXP3) gene leads to loss of Treg and/or their function $(14,15)$. A recent study used the combination of deep flow cytometric and single-cell RNA-seq profiling of Tregs and conventional CD4+ T cells from IPEX patients to identify gene signatures associated with IPEX (16). Using CRISPR-Cas9 genome editing, Goodwin et al. modified the FOXP3 gene in human hematopoietic stem cells to enhance the stability of FOXP3 expression and the suppressive capacity in Tregs (17). A similar gene editing approach in a mouse model resulted in the generation of "super Tregs" capable of resolving the severe inflammation in IPEX-like disorders by modulating the chromatin modifier Brg1 (18). Studies of monogenic disorders like IPEX could lead to new biomarkers and therapeutic strategies for managing polygenic autoimmune disorders. Dysregulation of the IL-1B, inflammasome-related proteins (NLR genes), and type-I interferon pathway represent additional mechanisms known to contribute to the loss of self-tolerance $(19,20)$, and need to be further studied to understand the role of dysregulated tolerance in human autoimmunity disease.

In contrast to monogenic diseases, autoimmune disorders such as systemic lupus erythematosus (SLE), rheumatoid arthritis (RA), multiple sclerosis (MS), and type 1 diabetes (T1D) are heritable diseases involving more than one gene and cell type in their etiology. Immune dysregulation observed in patients include enhanced activation of autoreactive Th1 and Th17 CD4 cells $(21,22)$, CD8 suppressor T lymphocytes targeting selfantigens in the CNS $(23,24)$, defective regulatory T cells (25-27), autoreactive B cells $(28,29)$, and aberrant T lymphocyte signaling and cytokine production (30-34). Given the complexity and heterogeneity associated with polygenic autoimmune disease, there is a need for better therapeutic approaches that specifically target pathogenic mechanisms. Understanding the specific cell types and functions dysregulated in autoimmune disease offers the potential for new drug targets and therapeutic approaches (35, 36). Tregs have been shown to be defective in the autoimmune disease settings, and ex vivo-expanded Tregs isolated from T1D patients have been used in phase I clinical trial as adoptive immunotherapy in T1D. In this trial expanded cells were found to retain their phenotype, TCR diversity, and functional capacity in patients for long periods (37). A transcriptomic study conducted in SLE patients showed that gene signatures associated with interferon signaling is significantly dysregulated (38), although current efforts targeting IFN in SLE have not been successful. A more recent study in this field profiled six major immune types in SLE patients by single-cell RNAseq and found a unique set of genes in monocytes, including two well-known immune modulators for SLE and RA therapeutics (TNFSF13B/BAFF: belimumab and IL1RN: anakinra, respectively) (39). A single cell transcriptomic study in Crohn's patients revealed a gene program associated with inflamed tissue, consisting of genes expressed by plasma cells, inflammatory mononuclear phagocytes, and activated T cells (40). These cutting-edge approaches are changing our appreciation of the complexity and heterogeneity of autoimmune disorders, and are helping to discover new therapeutic strategies and identifying new therapeutic biomarkers.

\section{COMPLEX GENETICS AND EPIGENETICS OF THE LOSS OF IMMUNOLOGIC TOLERANCE IN HUMANS}

Genetic predisposition and epigenetic modifications are implicated in the loss of tolerance and autoimmunity, and emerging genomic technologies are enabling comprehensive interrogation of genetic variants that contribute to autoimmune disease risk. Genome-wide association studies have implicated hundreds of loci in disease susceptibility, many of which are disease-specific. However, a number of risk loci are shared across multiple diseases, suggesting the involvement of common pathways associated with the loss of tolerance. The MHC locus is genetically associated with all autoimmune diseases $(41,42)$. Much of this linkage is thought to be driven by HLA coding polymorphisms that affect selfpeptide binding, however, this region contains over 200 genes, and high polymorphism and linkage disequilibrium across the locus presents technical challenges for identifying risk alleles and alternative causal genes. CTLA4 has been linked with T1D (43) and auto-antibody positive RA (44). This immunoglobulin superfamily member is expressed on the surface of conventional and regulatory $\mathrm{T}$ cells that transmits an 
inhibitory signal for $\mathrm{T}$ cell activation and strips costimulatory ligands from antigen presenting cells. A non-synonymous variant in PTPN22 was shown to be associated with many autoimmune diseases, including T1DM, RA, SLE and Graves disease (45-47). The risk variant in PTPN22 gene affects the binding of lymphoid phosphatase (LYP) to the signaling suppressor SRC kinase and ultimately affects the signaling pathways during $\mathrm{T}$ cell and $\mathrm{B}$ cell receptor response.

Several approaches have been used to map autoimmune disease variants to effector genes in recent years. The advent of the Illumina Infinium SNP Immunochip has helped to fine map many autoimmune GWAS loci including SLE. In a study of lupus, researchers used a random forest machine learning method to integrate Immunochip genotyping and $\mathrm{T}$ and $\mathrm{B}$ cell RNA-seq analysis from SLE patients and healthy control subjects, identifying three novel genes (ZNF804A, CDK1, and $M A N F$ ) that were not previously been associated with SLE or any other autoimmune disorder (48). To functionally validate the allele specific expression pattern of 3,000 genes identified by genotype data from the Immunochip, an eQTL analysis was performed in $\mathrm{B}$ and $\mathrm{T}$ cells from healthy donors which leads to the involvement of cis-regulatory SNPs in gene regulation. Conclusively, six SLE associated genes found to be regulated by cis-rSNPs were IKZF1, NCF2, IL12A and TNIP1 in B cells and $A N K 3$, and PHRF1 in T cells (48). The combination of machine learning and allele-specific transcriptome analysis represents a valuable tool for validation of target genes associated with disease risk and offers a functional follow-up strategy to test these molecular targets under clinical settings.

A growing body of work links epigenetic modifications in immune cells with autoimmune disease risk. Epigenetic processes like DNA methylation and histone modification contribute to the expression of genes associated with disease $(49,50)$, and characterization of epigenetic factors could provide deeper insight into the onset and progression of disease. An example is the association of SLE with perturbed DNA methylation, a process that influences expression of cytokines like IL-2, IFN-gamma, IL-4 and IL-13 (51-53), and the Treg transcription factor FOXP3 (54). Naïve T cells from SLE patients exhibit global hypomethylation due to decreased DNMT1 activity (55), with specific genes such as CD11a (ITGAL), perforin (PRF1), CD70 (TNFSF7), and CD40LG (TNFSF5) showing decreased DNA methylation (56). In addition, altered DNA methylation patterns at STAT3, IL6 and CXCL12 has been associated with RA pathogenesis (57), and expression of the epigenetic enzymes DNMT1, MBD3 and MBD4 were found to be decreased in systemic sclerosis patients. The latter was associated with increased expression of CD40L, CD11a, and $C D 70$ (58). As these epigenetic modifications alter gene expression programs of immune cells, epigenetics-based drugs and editing tools are emerging as a promising therapeutic option to restore healthy epigenetic landscapes under disease settings.

Integration of transcriptomic and epigenomic data with GWAS data provides a genome-scale view of the potential function of autoimmune risk variants in disease relevant celltypes. The Encyclopedia of DNA Elements (ENCODE) database has been used to corelate known genetic variants with histone $\mathrm{H} 3$ lysine 4 tri-methylation (H3K4me3) marks to identify cell types associated with particular autoimmune disease. Examples are studies that colocalized $31 \mathrm{RA}$-associated SNPs with H3K4me3 marks in CD4+ T cells (59), and colocalization of RNA-seq and ChIP-seq signals for the H3K4me1 and K3K27Ac enhancer marks in neutrophils and CD4+ T cells with JIA-associated variants in patients (60). Yuen et al. used publicly available ENCODE and Roadmap Epigenomics data generated in CD4+ $\mathrm{T}$ cells and $\mathrm{B}$ cells along with the ChIP-seq data generated in human neutrophils to examine the "epigenetic landscape" of SLE SNPs in a cell type-specific manner in adult immune cells (61). To identify whether immune disease variants regulate activation and differentiation, researchers profiled chromatin accessibility by ATAC-seq along with active enhancers and promoters by ChIPmentation-seq analysis in naïve and memory CD4+ T cells and macrophages. Using a newly developed statistical SNP enrichment method (CHEERS), the authors provided a comprehensive view of epigenetic mark enrichment at immune disease variants under specific activation and polarization conditions (62). The advent of single-cell genomics and gene editing technologies like CRISPR will allow functional validation of regulatory variants that influence immune tolerance and localize their effects to specific immune cell subsets.

\section{STATISTICAL LINKAGE OF AUTOIMMUNE RISK VARIANTS WITH TOLERANCE GENE EXPRESSION}

GWAS identifies large blocks $(10-1,000 \mathrm{~kb})$ of the genome that contain hundreds or thousands of SNPs, any of which could be causal (63), and colocalization studies like those described above have helped to identify potentially regulatory SNPs at GWAS loci. However, the vast majority of disease-associated SNPs and their associated epigenomic features are not located in gene promoters, and therefore the genes they regulate are not known. cis-eQTL (expression quantitative trait loci) analyses have been used to statistically link gene expression with SNP genotype. Most large eQTL studies so far have used peripheral blood expression data (64-67), and have linked $\sim 42 \%$ of autoimmune sentinel SNPs to expression of a gene at the locus. A number of studies have shown that causal SNPs (e.g., in celiac disease and rheumatoid arthritis) disrupt transcription factor binding sites (68-70) and massively parallel reporter assays have identified SNPs that affect the activity of regulatory elements (69). However, the majority of autoimmune loci lack eQTL support, likely due to use of data from undifferentiated and/or non-activated immune cells, as colocalization studies show that risk SNPs are enriched for functional marks mainly in stimulated and differentiated cell types (3). Using RNA-seq data collected from PBMC of 629 healthy patients, Ricano-Ponce et al. performed conditional ciseQTL mapping and implicated 233 causal genes (e.g., IL6, CXCR4, $B C L-X L, M Y C)$, including 53 long non-coding RNAs, from 120 loci associated with 14 autoimmune disorders (64). Another study linked 39 lupus-associated variants to genes through the integration of GWAS and eQTL data from TwinsUK microarray 
and exon-level RNA-seq cohort in lymphoblastoid cell lines. This study identified novel, SLE-associated splice variants and novel candidate SLE eGenes, including SOCS1, CSK, and the transcription factor IKZF2 involved in Treg stabilization during inflammatory responses $(71,72)$. Importantly, these studies showed that more than half of the associated genes were not those nearest to the candidate SNP.

\section{BIOPHYSICAL LINKAGE OF AUTOIMMUNE VARIANTS TO TOLERANCE GENES THROUGH CHROMOSOME CONFORMATION- BASED APPROACHES}

The human genome is organized in three-dimensional (3D) space in the nucleus into active and inactive compartments (73). Within active compartments, chromatin is organized into loops that can connect long-range regulatory elements with distant gene promoters. Recent high-throughput approaches for measuring the $3 \mathrm{D}$ structure of the genome in cells have provided new insights into global genome organization and the role of chromatin topology in genome function and dysfunction in health and disease. Two examples are studies by Jung et al. and Javierre et al. using a low-resolution Capture-HiC approaches to map the interactomes of $\sim 18,000$ human gene promoters in 27 human tissue/cell types (74), and $\sim 30,000$ promoter connectomes in 17 hematopoietic cell types (75). Genomic regions caught interacting with promoters were enriched for open chromatin, active histone marks, and disease-associated SNPs and eQTLs. Both studies were able to detect cell-type specific regulatory architectures, and the latter study also assembled a set of core genes connected to SNPs associated with multiple autoimmune disorders into an "autoimmune network" (75). Importantly, both studies found that less than $10 \%$ of disease-associated SNPs were connected to the nearest gene, further emphasizing that one cannot assume that risk SNPs (or other genomic features) regulate their nearest gene. Significant associations have been found between complex traits and gene deserts $(>500 \mathrm{~kb}$ of genomic region which either lack protein coding sequence or annotated gene) which suggests that disease causing SNPs can affect gene expression by altering transcription factor binding to long-distance regulatory elements (76-78). Thus, integrating complexity of 3D genome into functional validation of GWAS studies can help uncover new insights in autoimmune disease pathogenesis.

In a more recent study, $\mathrm{Su}$ et al. used the combination of high-resolution promoter-Capture-C and ATAC-seq to map regulatory SLE variants to their target genes (79). Importantly, this study focused on follicular T helper cells (TFH) 'caught in the act' of helping $B$ cells to produce antibodies in human tonsil. Unlike undifferentiated $\mathrm{T}$ cells or cell lines commonly used for these types of genome-scale studies, TFH play an active role in generating the pathogenic auto-antibodies characteristic of SLE, and represents a highly relevant target tissue for

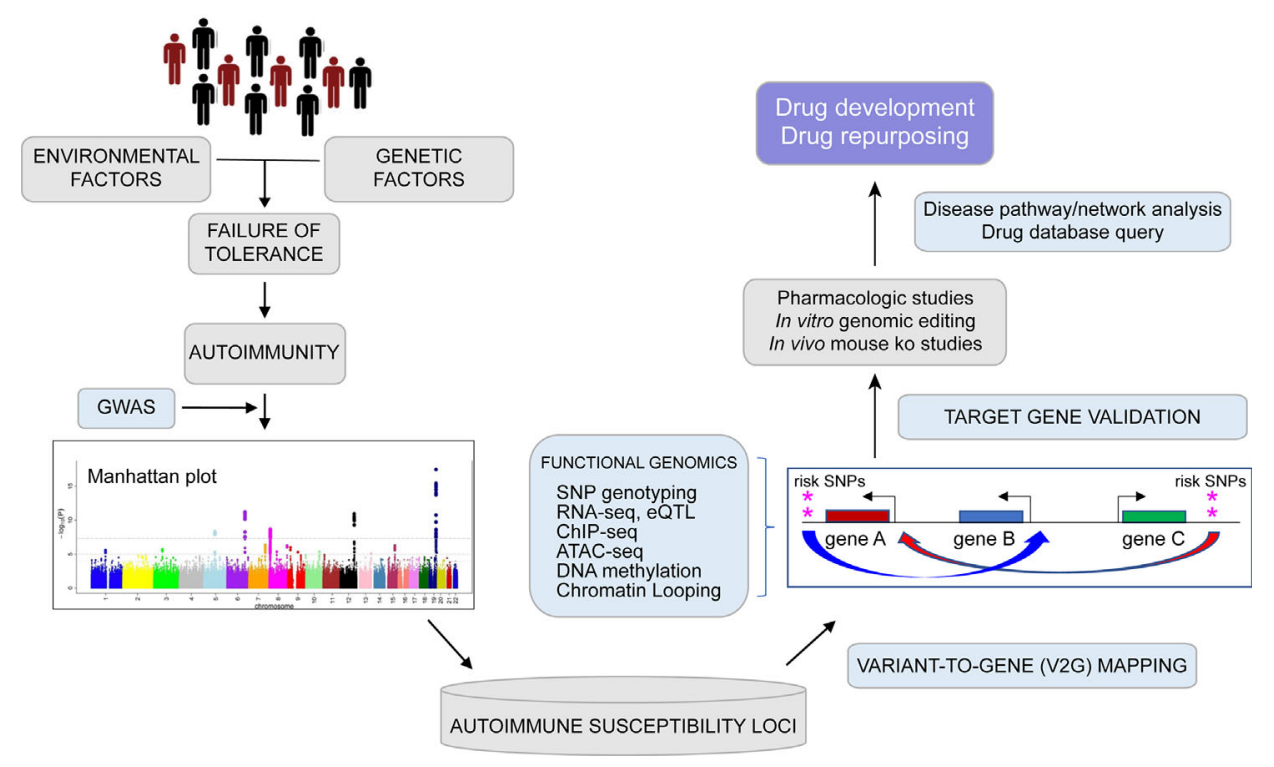

FIGURE 1 | From genome to function: Graphical depiction of a pipeline leveraging genetic and epigenetic datasets to connect auto-immune disease associated variants to their target genes with focus toward drug development and repurposing. Genome wide association studies (GWAS) can identify multiple common genetic variants that confer risk for various diseases (as shown by Manhattan plot) including auto-immune disorders, but which variants are causal and which genes are involved remains largely unknown. Expression quantitative trait locus (eQTL) studies, high-resolution analysis of epigenomic and spatial organization can connect potentially functional SNPs with expression of putative disease genes in relevant cell types. Disease pathway exploration and experimental validation may lead to drug development and repurposing efforts. 
functional genomic analyses. This study identified $\sim 400$ accessible SLE variants connected to a network of 330 genes enriched for high expression in TFH and roles in T cell differentiation, humoral immunity, systemic autoimmune disorders, rheumatic disease, and type 1 diabetes. Remarkably, the physical SLE SNP-gene linkages measured in this one cell type confirmed one out of three linkages established statistically (eQTL) in a prior study (8). CRISPR/ CAS9 genome editing validated novel SLE-associated regulatory elements that regulate TFH and SLE genes like BCL6, CXCR5, and IKAROS. A separate study combined the SNPs associated with 19 autoimmune diseases with cell-specific multi-omics approaches to develop an epigenetic weighted scoring method to evaluate the functionality of all noncoding autoimmune SNPs. The analysis also suggested long-range chromatin interactions between functional SNPs and distal target genes, highlighting the unique regulatory roles of noncoding SNPs associated with autoimmune diseases (80).

In addition to revealing the disease-associated regulatory architectures of known autoimmune genes, spatial maps of variant accessibility and gene connectivity in immune cell types can be used to identify novel genes involved in tolerance and autoimmunity. For example, the study by $\mathrm{Su}$ et al. highlighted a set of genes identified by virtue of their physical connection to SLE variants that had no prior known role in lupus or TFH function. Two of these genes encode the kinases HIPK1 and MINK1, and genetic or pharmacologic inhibition of their function in human TFH cells resulted in reduced secretion of IL21, a signature TFH cytokine required for class-switched antibody production by B cells. Targeting of HIPK1 in TFH reduced expression of the SLE genes PTPN22, IL6R, IL2R, $B A C H 2$, and PD1 (79). The coalescence of state-of-the-art, genome-scale 3D-omic data from relevant immune cell types holds promise to further our basic understanding of the mechanisms that control immune tolerance, and point to novel targets for therapeutic intervention to treat and/or prevent autoimmune and inflammatory disorders (Figure 1).

\section{REFERENCES}

1. Xing Y, Hogguist KA. T cell tolerance: Central and Peripheral. Cold Spring Harb Perspect Biol (2012) 4(6):1-15. doi: 10.1101/cshperspect.a006957

2. Ye J, Gillespie KM, Rodriguez S. Unravelling the Roles of Susceptibility Loci for Autoimmune Diseases in the Post-GWAS Era. Genes (Basel) (2018) 9:377. doi: 10.3390/genes 9080377

3. Farh KK, Marson A, Zhu J, Kleinewietfeld M, Housley WJ, Beik, et al. Genetic and epigenetic fine mapping of causal autoimmune disease variants. Nature (2015) 518(7539):337-43. doi: 10.1038/nature13835

4. Ernst J, Kheradpour P, Mikkelsen TS, Shoresh N, Ward LD, Epstein CB, et al. Mapping and analysis of chromatin state dynamics in nine human cell types. Nature (2011) 473(7345):43-9. doi: 10.1038/nature09906

5. Gerasimova A, Chavez L, Li B, Seumois G, Greenbaum J, Rao A, et al. Predicting cell types and genetic variations contributing to disease by combining GWAS and epigenetic data. PloS One (2013) 8(1):e54359. doi: 10.1371/journal.pone.0054359

6. Schmiedel BJ, Singh D, Madrigal A, Valdovino-Gonzalez AG, White BM, Zapardiel-Gonzalo J, et al. Impact of Genetic Polymorphisms on Human Immune Cell Gene Expression. Cell (2018) 175(6):1701-15. doi: 10.1016/ j.cell.2018.10.022

\section{CONCLUSION}

Detailed characterization of the functional effects of autoimmune disease-associated genetic variation on gene expression and immune cell function is of paramount significance to our understanding of immune tolerance. Interpreting SNP-trait associations requires integration of functional information from resources and repositories such as Genotype-Tissue Expression (GTEx), ENCODE, the Epigenomics Roadmap Project, and focused variant-to-gene (V2G) mapping studies like those described here. To overcome challenges like co-regulation of multiple genes and tissue heterogeneity, techniques must be fine-tuned to identify the most specific drug targets and biomarkers. Single-cell transcriptomic (scRNA-seq) and epigenomic (scATAC-seq) approaches offer the potential to dissect the contributions of cell, SNP, gene, and functional heterogeneity to immune disease. Tools for detecting and analyzing global genetic and epigenetic diversity are continuously evolving, and are on track to revolutionize our understanding of normal immune development and function, immune dysregulation and the breakdown of tolerance, and targets for new therapeutics to treat inflammation.

\section{AUTHOR CONTRIBUTIONS}

PM and AW conceptualized and wrote the manuscript. All authors contributed to the article and approved the submitted version.

\section{FUNDING}

AW is funded by NIH grants DK122586, AI123539, AI130115, and AI054643, and by the Center for Spatial and Functional Genomics at the Children's Hospital of Philadelphia.

7. Onengut-Gumuscu S, Chen WM, Burren O, Cooper NJ, Quinlan AR, Mychaleckyj JC, et al. Fine mapping of type 1 diabetes susceptibility loci and evidence for colocalization of causal variants with lymphoid gene enhancers. Nat Genet (2015) 47(4):381-6. doi: 10.1038/ng.3245

8. Bentham J, Morris DL, Graham DSC, Pinder CL, Tombleson P, Behrens TW, et al. Genetic association analyses implicate aberrant regulation of innate and adaptive immunity genes in the pathogenesis of systemic lupus erythematosus. Nat Genet (2015) 47(12):1457-64. doi: 10.1038/ng.3434

9. Akkaraju S, Ho WY, Leong D, Canaan K, Davis MM, Goodnow CC. A range of CD4 T cell tolerance: partial inactivation to organ-specific antigen allows nondestructive thyroiditis or insulitis. Immunity (1997) 7(2):255-71. doi: 10.1016/S1074-7613(00)80528-2

10. Klein L, Kyewski B. "Promiscuous" expression of tissue antigens in the thymus: a key to T-cell tolerance and autoimmunity? J Mol Med (Berl) (2000) 78(9):483-94. doi: 10.1007/s001090000146

11. Ohashi PS, Oehen S, Buerki K, Pircher H, Ohashi CT, Odermatt B, et al. Ablation of "tolerance" and induction of diabetes by virus infection in viral antigen transgenic mice. Cell (1991) 65(2):305-17. doi: 10.1016/0092-8674(91)90164-T

12. Janeway C. Immunobiology 5: The Immune System in Health and Disease. 5th ed. New York: Garland (2011). ISBN: OCLC 45708106. 
13. Romano M, Fanelli G, Albany CJ, Giganti G, Lombardi G. Past, Present, and Future of Regulatory T Cell Therapy in Transplantation and Autoimmunity. Front Immunol (2019) 10:43. doi: 10.3389/fimmu.2019.00043

14. Allenspach EJ, Finn LS, Rendi MH, Eken A, Singh AK, Oukka M, et al. Absence of functional fetal regulatory $\mathrm{T}$ cells in humans causes in utero organspecific autoimmunity. J Allergy Clin Immunol (2017) 140(2):616-9. doi: 10.1016/j.jaci.2017.02.017

15. Powell BR, Buist NR, Stenzel P. An X-linked syndrome of diarrhea, polyendocrinopathy, and fatal infection in infancy. J Pediatr (1982) 100 (5):731-7. doi: 10.1016/S0022-3476(82)80573-8

16. Zemmour D, Charbonnier L-M, Leon J, Six E, Keles S, Delville M, et al. Single cell analysis of FoxP3 deficiencies in humans and mice unmasks intrinsic and extrinsic CD4+ T cell perturbations. biorXiv (2020). doi: 10.1101/ 2020.07.06.189589

17. Goodwin M, Lee E, Lakshmanan U, Shipp S, Froessl L, Barzaghi F, et al. CRISPR-based gene editing enables FOXP3 gene repair in IPEX patient cells. Sci Adv (2020) 6(19):eaaz0571. doi: 10.1126/sciadv.aaz0571

18. Li Y, Chen Y, Mao S, Kaundal R, Jing Z, Chen Q, et al. In situ conversion of defective Treg into SuperTreg cells to treat advanced IPEX-like disorders in mice. Nat Commun (2020) 11:2781. doi: 10.1038/s41467-020-15836-2

19. Marson A, Housley WJ, Hafler DA. Genetic basis of autoimmunity. J Clin Invest (2015) 125(6):2234-41. doi: 10.1172/JCI78086

20. Manthiram K, Zhou Q, Aksentijevich I, Kastner DL. The monogenic autoinflammatory diseases define new pathways in human innate immunity and inflammation. Nat Immunol (2017) 18(8):832-42. doi: 10.1038/ni.3777

21. Nistala K, Adams S, Cambrook H, Ursu S, Olivito B, de Jager W, et al. Th17 plasticity in human autoimmune arthritis is driven by the inflammatory environment. Proc Natl Acad Sci USA (2010) 107:14751-6. doi: 10.1073/ pnas. 1003852107

22. Annunziato F, Cosmi L, Liotta F, Maggi E, Romagnani S, Annunziato F. Type $17 \mathrm{~T}$ helper cells-origins, features and possible roles in rheumatic disease. Nat Rev Rheumatol (2009) 5(6):325-31. doi: 10.1038/nrrheum.2009.80

23. Wagner CA, Roque PJ, Mileur TR, Liggitt D, Goverman JM. Myelin-specific CD8 + T cells exacerbate brain inflammation in CNS autoimmunity. J Clin Invest (2020) 130(1):203-13. doi: 10.1172/JCI132531

24. Salehi Z, Doosti R, Beheshti M, Janzamin E, Sahraian MA, Izad M. Differential frequency of $\mathrm{Cd} 8+\mathrm{T}$ cell subsets in Multiple Sclerosis pateints with various clinical patterns. PloS One (2016) 28:e0159565. doi: 10.1371/ journal.pone. 0159565

25. Dominguez-Villar M, Hafler DA. Regulatory $\mathrm{T}$ cells in autoimmune disease. Nat Immunol (2018) 19(7):665-73. doi: 10.1038/s41590-018-0120-4

26. Sumida T, Lincoln MR, Ukeje CM, Rodriguez DM, Akazawa H, Noda T, et al. Activated beta-catenin in Foxp3(+) regulatory T cells links inflammatory environments to autoimmunity. Nat Immunol (2018) 19(12):1391-402. doi: 10.1038/s41590-018-0236-6

27. Spence A, Purtha W, Tam J, Dong S, Kim Y, Ju CH, et al. Revealing the specificity of regulatory $\mathrm{T}$ cells in murine autoimmune diabetes. Proc Natl Acad Sci USA (2018) 115(20):5265-70. doi: 10.1073/pnas.1715590115

28. Jenks SA, Cashman KS, Zumaquero E, Marigorta UM, Patel AV, Wang X, et al. Distinct Effector B Cells Induced by Unregulated Toll-like Receptor 7 Contribute to Pathogenic Responses in Systemic Lupus Erythematosus. Immunity (2018) 49(4):725-39. doi: 10.1016/j.immuni.2018.08.015

29. Sang A, Danhorn T, Peterson JN, Rankin AL, O'Connor BP, Leach SM, et al. Innate and adaptive signals enhance differentiation and expansion of dualantibody autoreactive B cells in lupus. Nat Commun (2018) 9(1):3973. doi: 10.1038/s41467-018-06293-z

30. Krishnan S, Farber DL, Tsokos GC. T cell rewiring in differentiation and disease. J Immunol (2003) 171(7):3325-31. doi: 10.4049/jimmunol.171.7.3325

31. Tenbrock K, Juang YT, Kyttaris VC, Tsokos GC. Altered signal transduction in SLE T cells. Rheumatology (Oxford) (2007) 46(10):1525-30. doi: 10.1093/ rheumatology/kem154

32. Dolff S, Scharpenberg C, Specker C, Kribben A, Witzke O, Winde B. Il-22 production of effector Cd4+ T cells is altered in SLE patients. Eur J Med Res (2019) 24:24. doi: 10.1186/s40001-019-0385-6

33. Ohl $\mathrm{K}$, Tenbrock $\mathrm{K}$. Inflammatory cytokines in systemic lupus erythematosus. J BioMed Biotechnol (2011) 2011:432595. doi: 10.1155/2011/432595

34. Nambiar MP, Fisher CU, Warke VG, Krishnan S, Mitchell JP, Delaney N, et al. Reconstitution of deficient $\mathrm{T}$ cell receptor zeta chain restores $\mathrm{T}$ cell signaling and augments $\mathrm{T}$ cell receptor/CD3-induced interleukin-2 production in patients with systemic lupus erythematosus. Arthritis Rheum (2003) 48(7):1948-55. doi: 10.1002/art.11072

35. Alvarez-Sanchez N, Cruz-Chamorro I, Diaz-Sanchez M, Lardone PJ, Guerrero JM, Carrillo-Vico AN. Peripheral CD39-expressing T regulatory cells are increased and associated with relapsing-remitting multiple sclerosis in relapsing patients. Sci Rep (2019) 9(1):2302. doi: 10.1038/s41598-01938897-w

36. von Spee-Mayer C, Siegert E, Abdirama D, Rose A, Klaus A, Alexander T, et al. Low-dose interleukin-2 selectively corrects regulatory $\mathrm{T}$ cell defects in patients with systemic lupus erythematosus. Ann Rheum Dis (2016) 75 (7):1407-15. doi: 10.1136/annrheumdis-2015-207776

37. Bluestone JA, Trotta E, Xu D. Type 1 diabetes immunotherapy using polyclonal regulatory T cells. Sci Transl Med (2015) 7:315. doi: 10.1126/ scitranslmed.aad4134

38. Rai R, Chauhan SK, Singh VV, Rai M, Rai G. RNA-seq Analysis Reveals Unique Transcriptome Signatures in Systemic Lupus Erythematosus Patients with Distinct Autoantibody Specificities. PloS One (2016) 11(11):e0166312. doi: 10.1371/journal.pone.0166312

39. Panwar B, et al. Integrative transcriptomic analysis of SLE reveals IFN-driven cross-talk between immune cells. bioRxiv (2020). doi: 10.1101/ 2020.04.27.065227

40. Martin JC, Chang C, Boschetti G, Ungaro R, Giri M, Grout JA, et al. SingleCell Analysis of Crohn's Disease Lesions Identifies a Pathogenic Cellular Module Associated with Resistance to Anti-TNF Therapy. Cell (2019) 178:1493-508. doi: 10.1016/j.cell.2019.08.008

41. Gaffney PM, Kearns GM, Shark KB, Ortmann WA, Selby SA, Malmgren ML, et al. A genome-wide search for susceptibility genes in human systemic lupus erythematosus sib-pair families. Proc Natl Acad Sci USA (1998) 95(25):148759. doi: 10.1073/pnas.95.25.14875

42. Rich SS, Weitkamp LR, Barbosa J. Genetic heterogeneity of insulin-dependent (type I) diabetes mellitus: evidence from a study of extended haplotypes. Am J Hum Genet (1984) 36(5):1015-23.

43. Nistico L, Buzzetti R, Pritchard LE, Van der Auwera B, Giovannini C, Bosi E, et al. The CTLA-4 gene region of chromosome $2 \mathrm{q} 33$ is linked to, and associated with, type 1 diabetes. Belgian Diabetes Registry. Hum Mol Genet (1996) 5:1075-80. doi: $10.1093 / \mathrm{hmg} / 5.7 .1075$

44. Plenge RM, Padyukov L, Remmers EF, Purcell S, Lee AT, Karlson EW, et al. Replication of putative candidate-gene associations with rheumatoid arthritis in $>4,000$ samples from North America and Sweden: association of susceptibility with PTPN22, CTLA4, and PADI4. Am J Hum Genet (2005) 77:1044-60. doi: 10.1086/498651

45. Begovich AB, Carlton VE, Honigberg LA, Schrodi SJ, Chokkalingam AP, Alexander $\mathrm{HC}$, et al. A missense single-nucleotide polymorphism in a gene encoding a protein tyrosine phosphatase (PTPN22) is associated with rheumatoid arthritis. Am J Hum Genet (2004) 75:330-7. doi: 10.1086/422827

46. Velaga MR, Wilson V, Jennings CE, Owen CJ, Herington S, Donaldson PT, et al. The codon 620 tryptophan allele of the lymphoid tyrosine phosphatase (LYP) gene is a major determinant of Graves' disease. J Clin Endocrinol Metab (2004) 89:5862-5. doi: 10.1210/jc.2004-1108

47. Bottini N, Musumeci L, Alonso A, Rahmouni S, Nika K, Rostamkhani M, et al. A functional variant of lymphoid tyrosine phosphatase is associated with type I diabetes. Nat Genet (2004) 36:337-8. doi: 10.1038/ng1323

48. Almlof JC, Alexsson A, Imgenberg-Kreuz J, Sylwan L, Backlin C, Leonard D, et al. Novel risk genes for systemic lupus erythematosus predicted by random forest classification. Sci Rep (2017) 7(1):6236. doi: 10.1038/s41598017-06516-1

49. Issuree PD, Day K, Au C, Raviram R, Zappile P, Skok JA, et al. Stage-specific epigenetic regulation of $\mathrm{CD} 4$ expression by coordinated enhancer elements during T cell development. Nat Commun (2018) 9(1):3594. doi: 10.1038/ s41467-018-05834-w

50. Kanno Y, Vahedi G, Hirahara K, Singleton K, O'Shea JJ. Transcriptional and epigenetic control of $\mathrm{T}$ helper cell specification: molecular mechanisms underlying commitment and plasticity. Annu Rev Immunol (2012) 30:70731. doi: 10.1146/annurev-immunol-020711-075058

51. Thomas RM, Gamper JC, Ladle BH, Powell JD, Wells AD. De novo DNA methylation is required to restrict $\mathrm{T}$ helper lineage plasticity. J Biol Chem (2012) 287:22900-9. doi: 10.1074/jbc.M111.312785 
52. Lee PP, Fitzpatrick DR, Beard C, Jessup HK, Lehar S, Makar KW, et al. A critical role for Dnmt1 and DNA methylation in T cell development, function, and survival. Immunity (2001) 15(5):763-74. doi: 10.1016/S1074-7613(01)00227-8

53. Bruniquel D, Schwartz RH. Selective, stable demethylation of the interleukin-2 gene enhances transcription by an active process. Nat Immunol (2003) 4 (3):235-40. doi: 10.1038/ni887

54. Morikawa H, Sakaguchi S. Genetic and epigenetic basis of Treg cell development and function: from a FoxP3-centered view to an epigenomedefined view of natural Treg cells. Immunol Rev (2014) 259(1):192-205. doi: 10.1111/imr.12174

55. Chen SH, Lv QL, Hu L, Peng MJ, Wang GH, Sun B. DNA methylation alterations in the pathogenesis of lupus. Clin Exp Immunol (2017) 187(2):18592. doi: $10.1111 /$ cei.12877

56. Richardson B. Primer: epigenetics of autoimmunity. Nat Clin Pract Rheumatol (2007) 3(9):521-7. doi: 10.1038/ncprheum0573

57. Ciechomska M, Roszkowski L, Maslinski W. DNA methylation as a future therapeutic and diagnostic target in Rheumatoid Arthritis. Cells (2019) 8:953. doi: 10.3390/cells8090953

58. Mazzone R, Zwergel C, Artico M, Taurone S, Ralli M, Greco A, et al. The emerging role of epigenetics in human autoimmune disorders. Clin Epigenet (2019) 11(1):34. doi: 10.1186/s13148-019-0632-2

59. Trynka G, Sandor C, Han B, Xu H, Stranger BE, Liu XS, et al. Chromatin marks identify critical cell types for fine mapping complex trait variants. Nat Genet (2013) 45(2):124-30. doi: 10.1038/ng.2504

60. Jiang K, Zhu L, Buck MJ, Chen Y, Carrier B, Tao L, et al. Disease-Associated SNPs From non-Coding Regions in Juvenile Idiopathic Arthritis Are Located Within or Adjacent to Functional Genomic Elements of Human Neutrophils and CD4+ T Cells. Arthritis Rheumatol (2015) 67:1966-77. doi: 10.1002/art.39135

61. Hui-Yuen JS, Zhu L, Wong LP, Jiang K, Chen Y, Liu T, et al. Chromatin landscapes and genetic risk in systemic lupus. Arthritis Res Ther (2016) 18 (1):281. doi: 10.1186/s13075-016-1169-9

62. Soskic B, Cano-Gamez E, Smyth DJ, Rowan WC, Nakic N, Esparza-Gordillo J, et al. Chromatin activity at GWAS loci identifies T cell states driving complex immune diseases. Nat Genet (2019) 51:1486-1493(2019). doi: 10.1038/ s41588-019-0493-9

63. Visscher PM, Wray NR, Zhang Q, Sklar P, McCarthy MI, Brown MA, et al. 10 Years of GWAS Discovery: Biology, Function, and Translation. Am J Hum Genet (2017) 101(1):5-22. doi: 10.1016/j.ajhg.2017.06.005

64. Ricano-Ponce I, Zhernakova DV, Deelen P, Luo O, Li X, Isaacs A, et al. Refined mapping of autoimmune disease associated genetic variants with gene expression suggests an important role for non-coding RNAs. J Autoimmun (2016) 68:62-74. doi: 10.1016/j.jaut.2016.01.002

65. Kumar V, Gutierrez-Achury J, Kanduri K, Almeida R, Hrdlickova B, Zhernakova DV, et al. Systematic annotation of celiac disease loci refines pathological pathways and suggests a genetic explanation for increased interferon-gamma levels. Hum Mol Genet (2015) 24(2):397-409. doi: $10.1093 / \mathrm{hmg} / \mathrm{ddu} 453$

66. Lappalainen T, Sammeth M, Friedlander MR, t Hoen PA, Monlong J, Rivas MA, et al. Transcriptome and genome sequencing uncovers functional variation in humans. Nature (2013) 501(7468):506-11. doi: 10.1038/nature12531

67. Zhernakova DV, Deelen P, Vermaat M, van Iterson M, van Galen M, Arindrarto $\mathrm{W}$, et al. Identification of context-dependent expression quantitative trait loci in whole blood. Nat Genet (2017) 49(1):139-45. doi: 10.1038/ng.3737

68. Soderquest K, Hertweck A, Giambartolomei C, Henderson S, Mohamed R, Goldberg R, et al. Genetic variants alter T-bet binding and gene expression in mucosal inflammatory disease. PloS Genet (2017) 13(2):e1006587. doi: 10.1371/journal.pgen.1006587

69. Tewhey R, Kotliar D, Park DS, Liu B, Winnicki S, Reilly SK, et al. Direct identification of hundreds of expression-modulating variants using a multiplexed reporter assay. Cell (2016) 165:1519-29. doi: 10.1016/ j.cell.2016.04.027

70. Maurano MT, Humbert R, Rynes E, Thurman RE, Haugen E, Wang H, et al. Systematic localization of common disease-associated variation in regulatory DNA. Science (2012) 337(6099):1190-5. doi: 10.1126/science.1222794

71. Odhams CA, Cortini A, Chen L, Roberts AL, Vinuela A, Buil A, et al. Mapping eQTLs with RNA-seq reveals novel susceptibility genes, non-coding RNAs and alternative-splicing events in systemic lupus erythematosus. Hum $\mathrm{Mol}$ Genet (2017) 26(5):1003-17. doi: 10.1093/hmg/ddw417

72. Kim HJ, Barnitz RA, Kreslavsky T, Brown FD, Moffett H, Lemieux ME, et al. Stable inhibitory activity of regulatory $\mathrm{T}$ cells requires the transcription factor Helios. Science (2015) 350(6258):334-9. doi: 10.1126/science.aad0616

73. Dekker J, Mirny L. The 3D genome as a moderator of chromosomal communication. Cell (2016) 164(1):1110-21. doi: 10.1016/j.cell.2016.02.007

74. Jung I, Schmitt A, Diao Y, Lee AJ, Liu T, Yang D, et al. A compendium of promoter-centered long-range chromatin interactions in the human genome. Nat Genet (2019) 51:1442-9. doi: 10.1038/s41588-019-0494-8

75. Javierre BM, Burren OS, Wilder SP, Kreuzhuber R, Hill SM, Sewitz S, et al. Lineage-Specific Genome Architecture Links Enhancers and Non-coding Disease Variants to Target Gene Promoters. Cell (2016) 167(5):1369-84. doi: 10.1016/j.cell.2016.09.037

76. Schierding W, Cutfield WS, O'Sullivan JM. The missing story behind Genome Wide Association Studies: single nucleotide polymorphisms in gene deserts have a story to tell. Front Genet (2014) 5:39. doi: 10.3389/ fgene.2014.00039

77. Li MJ, Wang LY, Xia Z, Sham PC, Wang J. GWAS3D: detecting human regulatory variants by integrative analysis of genome-wide associations, chromosome interactions and histone modifications. Nucleic Acids Res (2013) 41:W150-8. doi: 10.1093/nar/gkt456

78. Fu Y, Tessneer KL, Li C, Gaffney PM. From association to mechanism in complex disease genetics: the role of the 3D genome. Arthritis Res Ther (2018) 20:216. doi: 10.1186/s13075-018-1721-x

79. Su C, Johnson ME, Torres A, Thomas RT, Manduchi E, Sharma P, et al. Mapping effector genes at lupus GWAS loci using promoter Capture-C in follicular helper T cells. Nat Commun (2020) 11:3294. doi: 10.1038/s41467020-17089-5

80. Chen XF, Guo M-R, Duan Y-Y, Jiang F, Wu H, Dong S-S, et al. Multi-omics dissection of molecular regulatory mechanisms underlying autoimmuneassociated noncoding SNPs. JCI Insight (2020) 5(17). doi: 10.1172/ jci.insight. 136477

Conflict of Interest: The authors declare that the research was conducted in the absence of any commercial or financial relationships that could be construed as a potential conflict of interest.

Copyright (c) 2021 Mehra and Wells. This is an open-access article distributed under the terms of the Creative Commons Attribution License (CC BY). The use, distribution or reproduction in other forums is permitted, provided the original author(s) and the copyright owner(s) are credited and that the original publication in this journal is cited, in accordance with accepted academic practice. No use, distribution or reproduction is permitted which does not comply with these terms. 